
\title{
28 Research Suare \\ Extracellular HMGB1 Interacts with RAGE and Promotes Chemoresistance in Acute Leukemia Cells
}

\section{Weixin Lai ( $\sim 2529120736 @ q q . c o m$ )}

Sun Yat-Sen University 2nd Affiliated Hospital: Sun Yat-Sen Memorial Hospital https://orcid.org/00000001-6828-014X

\section{QIAN KONG}

Third Affiliated Hospital of Sun Yat-Sen University

\section{HAN CHEN}

Sun Yat-Sen University 2nd Affiliated Hospital: Sun Yat-Sen Memorial Hospital

\section{XINYU LI}

Sun Yat-Sen University 2nd Affiliated Hospital: Sun Yat-Sen Memorial Hospital

\section{YUNYAO LI}

Sun Yat-Sen University 2nd Affiliated Hospital: Sun Yat-Sen Memorial Hospital

\section{JIANPEI FANG}

Sun Yat-Sen University 2nd Affiliated Hospital: Sun Yat-Sen Memorial Hospital

\section{Research Article}

Keywords: Acute leukemia, HMGB1, RAGE, autophagy, apoptosis, drug efflux protein, drug -resistance.

Posted Date: March 31st, 2021

DOI: https://doi.org/10.21203/rs.3.rs-349396/v1

License: (c) (1) This work is licensed under a Creative Commons Attribution 4.0 International License.

Read Full License

Version of Record: A version of this preprint was published at Cancer Cell International on December 1st, 2021. See the published version at https://doi.org/10.1186/s12935-021-02387-9. 


\section{Abstract}

Background Nowadays, acute leukemia(AL) among children has a more favorable outcome, yet some of them get refractory or relapse mainly due to drug resistance. High-mobility group box 1 (HMGB1) has been proven to have a important role in drug resistance via upregulation of autophagy after chemotherapy treatment in acute leukemia. However, the mechanism how extracellular HMGB1 acts on AL cells and leads to chemoresistance remains elusive. Method CCK8 was used to examine the toxicity of chemotherapeutic drug.Western blot and mRFP-GFP-LC3 adenoviral particles as well as transmission electron microscopy were used to detect the autophagy flux. Elisa was performed to detect the release of HMGB1.Western blot and flow cytometry were applied to evaluate the apoptosis. qPCR and western blot were conducted to detect the expression of drug efflux protein. Lentivirus infection was applied to knock down RAGE. In addition, T-ALL NOD/SCID mice xenograft model was used to observe the effect of inhibiting HMGB1/RAGE axis. Results We found that extracellular HMGB1 do upregulate autophagy and in the meantime downregulate apoptosis, primarily through interaction with receptor for advanced glycation end products (RAGE). Suppression of RAGE by RNA interference alleviated the level of autophagy and enhanced apoptosis. What's more, HMGB1/RAGE induced autophagy was associated with the activation of ERK $1 / 2$ and decreased phosphorylation of mammalian target of rapamycin (mTOR), while HMGB1/RAGE limited apoptosis in a Bcl-2-regulated way mediated by P53. On the other hand, we found that HMGB1/RAGE activated the NF-KB pathway and promoted the expression of Pglycation protein(P-gp) as well as multidrug resistance-associated protein(MRP), both are ATP-binding cassette transporters. In vivo experiment, we found that blocking HMGB1/RAGE axis do have a mild pathological condition and a better survival in T-ALL mice. Conclusion HMGB1/RAGE have a important role in drug resistance after chemotherapy treatment, mainly by regulating autophagy and apoptosis as well as promoting the expression of drug efflux protein such as P-gp and MRP. HMGB1/RAGE might be a promising target to cure $\mathrm{AL}$, especially for those met with relapse and refractory.

\section{Introduction}

Acute leukemia, the most common cancer happened in children, is originated from the abnormal proliferation and differentiation of hematopoietic stem cell(HSC), which results in the disturbance and deterioration of the function of bone marrow ${ }^{[1]}$. Acute leukemia has two main subtypes: acute lymphoblastic leukemia(ALL) and acute myeloid leukemia(AML), classified by their affected lineage $\mathrm{e}^{[2,3]}$. Nowadays, with the advancement in supportive care, chemotherapy treatment and risk stratification, treatment of acute leukemia happened in children has gained great achievement, with an overall cure rate of more than $70 \%$. Particularly, the 5 -year survival rate of ALL is above $85 \%$ in developed countries. However, in China,the 5 -year event-free survival rate of ALL is about $82 \%$, and the cumulative risk of relapse was $24.5 \%$ at 10 years, which often confers worse prognosis ${ }^{[4,5]}$. The reason of refractory and relapse often arises from the acquisition of drug resistance. Furthermore, the dysregulation of autophagy and apoptosis, as well as the the upregulated expression of ATP-binding cassette transporters (transporter-mediated drug efflux), contribute greatly to the occurrence of drug resistance ${ }^{[6]}$. 
Autophagy, as a conserved cellular recycling process, plays an important role in maintaining homeostasis and stress adaptation. When cells undergo hypoxia, stress, starvation, chemotherapy treatment, autophagy is upregulated to clear the damaged organelle, degrade cellular components to increase nutrient availability and reduce toxic wastes, which promotes the survival of cancer cells ${ }^{[7,8]}$. While apoptosis, also known as programmed cell death, also acts as a defense mechanism to eliminate damaged cells or cells with harmful mutations. And the dysregulation and evasion of apoptosis is common and important aspect in the development of cancer, which is considered as the hallmark of cancer $^{[9,10]}$. The role of autophagy and apoptosis in chemotherapy treatment is complicated and depends on the progression of tumor, drug properties and so on. It is said that they are double-edge sword in treating cancers ${ }^{[11-13]}$. To clarify the relationship of autophagy and apoptosis in different stages of disease is beneficial for us to cure leukemia more reasonably.

Currently, a growing body of literature pointed out the central role of high-mobility group protein 1 (HMGB1) in regulating autophagy and apoptosis, which contributes greatly to the development of chemotherapeutic resistance ${ }^{[14-19]}$. HMGB1 is a highly conserved nuclear protein that has been proven to be upregulated in various kinds of cancers. Studies demonstrated that HMGB1 was over-expressed in three leukemia cell lines $₫ \mathrm{~K} 562, \mathrm{HL} 60$ and Jurkat) and was correlated to the stage of leukemia ${ }^{[20,21]}$. And it is reported that the expression of HMGB1 in primary and relapsed leukemia was much higher than healthy people and patients with complete remission ${ }^{[22]}$. HMGB1 is mainly located in nucleus in quiescent conditions. However, in response to stress or chemotherapy treatments, HMGB1 can translocate into the cytoplasm and excrete into extracellular matrix via post-translational modification such as acetylation, poly ADP-ribosylation and so on. Study showed that Poly (ADP-ribosylation) of HMGB1 facilitated its acetylation and promoted HMGB1 translocation-associated chemotherapy-induced autophagy in leukemia cells in which acetylation of HMGB1 is the most crucial process ${ }^{[23]}$.In different parts of cell, HMGB1 has different functions. In nucleus, HMGB1 acts as DNA chaperone, participating in DNA repairs and sustaining the stability of chromosomal. What's more, it can modulate the transcription of some genes such as P53 and NF-KB. Meanwhile, Tang et al proved that HMGB1 could promote the transcription of HSP27 and then enhanced autophagy through Pink1/Parkin pathway. In cytoplasm, HMGB1 dislocates Beclin1-Bcl2 and contributes to the formation of Beclin1/PI3K-III complex to induce autuphagy, mainly via MAPK/ERK signaling pathway. What's more, it was demonstrated that HMGB1 can promote autophagy by activating PI3K/AKT/mTORC1 pathway. Extracellular HMGB1 also have been found to upregulate autophagy by activating AMPK/ERK pathway and AMPK/mTOR signaling pathway. And receptor for advanced glycation end products (RAGE) was involved in this process ${ }^{[20,24-27]}$.

RAGE is an evolutionarily single transmembrane member of the immunoglobulin superfamily. RAGE binds to an array of structurally diverse ligands, which include advanced glycation endproducts, S100/calgranulin family, extracellular HMGB1 and so on, acting as a pattern recognition receptor (PRR) and is expressed on cells of different origins performing different functions. RAGE is the first reported receptor of HMGB1 with high affinity. ${ }^{[28-31]}$ Studies showed that RAGE played an indispensable role in HMGB1 induced autophagy. And a number of studies demonstrated that RAGE was involved in the 
proliferation, differentiation, metastasis of tumor cells. What's more, RAGE can alleviate the ROS level, sustain autophagy and suppress apoptosis in pancreatic tumor cells ${ }^{[31-34]}$. However, the role of HMGB1/RAGE axis have not been studied in AL.

Transporter-mediated drug efflux, such as P-gp and MRP, also have great association with the occurrence of drug resistance. Both P-gp and MRP are belong to ATP-binding cassette transporters, encoded by $A B C B 1 / A B C C 1$. And they are able to eject a variety of substrates from cytoplasm to extracelluar matrix, including chemotherapeutic agents ${ }^{[35]}$. A great number of studies asserted that drug efflux proteins have a close relationship with drug resistance. And it was believed that anthracycline agents such as adriamycin(ADM), daunorubicin(DNR), are prone to induce drug resistance by upregulating the expression of ATP-binding cassette transporters ${ }^{[36-38]}$. Furthermore, evidences showed that extracelluar HMGB1 could upregulate the expression of P-gp and MRP in gastric adenocarcinoma cells. In status epilepticus rat brains, researchers found that extracelluar HMGB1 regulated P-gp expression via RAGE/NF-KB signaling pathway. On the other hand, a number of studies showed that NF-KB was the downstream of HMGB $1^{[39,40]}$. However,it still remain unknown whether HMGB1/RAGE has a relationship with NF-KB and the upregulation of ATP-binding cassette transporters in AL.

We have previously demonstrated that ULK1/2-ATG13-FIP200 complex, one of the critical effectors of autophagosome formation, acting as upstream of HMGB1-Beclin1, played a critical role in autophagy induced by DNR. What's more, we found that poly (ADP-ribosylation) of HMGB1 facilitated its acetylation and promoted HMGB1 translocation-associated chemotherapy-induced autophagy in leukemia cells. In this study,we show that extracelluar HMGB1 interacts with RAGE to sustain AL cells survival by promoting autophagy and decreasing apoptosis. What'more, HMGB1/RAGE enhanced the expression of P-gp and MRP via activating the NF-KB pathway. These findings suggest that HMGB1/RAGE promotes tumor cell survival after chemotherapy treatments. And we provides some evidences for the development of novel clinical approaches targeting the HMGB1/RAGE pathway.

\section{Materials And Methods}

\section{Subjects and cell culture.}

The human acute lymphoblastic leukemia cell line Jurkat and the human acute myeloid leukemia cell line HL-60 were purchased from the Type Culture Collection of the Chinese Academy of Sciences; Both Jurkat and HL-60 cells were cultured in RPMI-1640 (Gibco; Thermo Fisher Scientific, Inc.) with 10\% fetal bovine serum (Gibco; Thermo Fisher Scientific, Inc.) in a humidified incubator with 5\% CO2 and 95\% air.

\section{Antibodies and reagents.}

Antibodies specific for GAPDH(catalogue no. 5172S), $\beta$-actin(catalogue no. 3700S), HMGB1 (catalogue no. 6893S), LC3 (catalogue no. 3868S), Bax(catalogue no. 5023S), Bcl 2(catalogue no. 15071S), NF-kB p65 (catalogue no. 8242S), phosphorylated p65(catalogue no. 3033S), erk(catalogue no. 9102S), phosphorylated erk(catalogue no. H9539), were obtained from Cell Signaling Technology. Antibodies 
specific for P62 (catalogue no.ab56416), RAGE(catalogue no. ab3611), P-gp(catalogue no. ab170904), Cleaved caspase 3(catalogue no. ab32042), phosphorylated mTOR(catalogue no. ab137133), mTOR(catalogue no.ab134903), phosphorylated P53(catalogue no.ab33889), P53((catalogue no.ab32389), PUMA(catalogue no.ab33906) were obtained from Abcam. Antibodies specific for MRP(catalogue no.abs116285) was obtained from Absin Bioscience, Inc. Antibodies specific for PARP(catalogue no.A0942) was obtained from ABclonal Technology,Inc. Antibodies that mentioned above were diluted to 1:1000 in 5\% BSA to detect the target protein. The secondary antibodies, including sheep anti-mouse IgG-HRP (catalogue no. RM3001), sheep anti-rabbit IgG-HRP (catalogue no. RM3002) were obtained from Beijing Ray Antibody Biotech. They were diluted to 1:5000 in TBST(1\% Tween 20). Recombinant Human HMGB1/High mobility group protein B1 (catalogue no.abs04694) was obtained from Absin Bioscience, Inc. Adriamycin(ADM) was purchased from MedChemExpress.

\section{Drug treatment}

Both Jurkat and HL-60 cells were treated with different concentrations of adriamycin(ADM) $ه 0,0.05,0.1$, $0.2,0.4,0.8 \mu \mathrm{M})$ for $24 \mathrm{~h}$ to test the cell viability. What's more, we pretreated cells with extracelluar $\operatorname{HMGB} 1(0,25,50,100 \mathrm{ng} / \mathrm{mL})$ for $24 \mathrm{~h}$ and then they were treated with ADM $(0.4 \mu \mathrm{M})$. In the subsequent experiments, both Jurkat and HL-60 cells were transfected with lentivirus to knock down the expression of RAGE. Then we pretreated them with extracelluar HMGB1 $(50 \mathrm{ng} / \mathrm{mL})$ for $24 \mathrm{~h}$, and then cells were incubated with $\operatorname{ADM}(0.2 \mu \mathrm{M})$ for another $24 \mathrm{~h}$. To figure out the role of P53, ERK2/1, mTOR and NF-KB in HMGB1/RAGE downstream signals, we then added their small molecular inhibitor piriffin- $\beta$, PD98059, Rapamycin and pyrrolidinedithiocarbamate ammonium(PDTC) into cells to inhibit those pathways with or without HMGB1. Then they were treated with $A D M(0.4 \mu \mathrm{M})$ for another $24 \mathrm{~h}$.

\section{Cell viability assay}

Both Jurkat and HL-60 cells were seeded in $96-$ well plates at a density of $3 \times 10^{4} / \mathrm{ml}$, after treated with different concentration of chemotherapeutic drug for $24 \mathrm{~h}, 10 \mu \mathrm{L}$ of the Cell Counting Kit-8 solution هDojindo Molecular Technologies, Inc.『were added into wells and incubated for $2 \mathrm{~h}$. Then the absorbance at $450 \mathrm{~nm}$ was measured in a microplate reader.

\section{Quantitative real-time PCR (RT-qPCR)}

Total RNA was isolated from both Jurkat and HL-60 cells using RNA-Quick Purification Kit (Yishan Biotech Inc.) and then reverse-transcribed into CDNA. The sequences of primers used were as follows:GAPDH: forward, 5'-ATGACTCTACCCACGGCAAG-3' and reverse, 5'-TACTCAGCACCAGCATCACC -3'; for HMGB1: forward, 5'-CTGTCCATTGGTGATGTTGC-3' and reverse, 5'-CTGATAGCCTGCTCCAGGTC-3'; for RAGE: forward, 5'-GAATCCTCCCCAATGGTTCA -3' and reverse, 5'-GCCCGACACCGGAAAGT -3'; for ABCB1: forward,5'-TGATTGCATTTGGAGGACAA -3' and reverse, 5'-CCAGAAGGCCAGAGCATAAG -3'; for ABCC1: forward, 5'-GGTTTATAGTAGGATTTACACGTGGTTG -3'and reverse, 5'-

AAGATAGTATCTTTGCCCAGACAGC -3'. Reactions were carried out in a Roche Light Cycler 96 system (Roche Diagnostics GmbH, Mannheim, Germany) with a SYBR 
Premix ExTaq kit (Takara Bio Inc., Otsu, Shiga, Japan). Data were normalized to GAPDH expression and analyzed with the $2^{-\Delta \Delta C t}$ method,.

\section{Western blot analysis.}

The two cell lines were subjected to the aforementioned different ADM concentrations and other chemical agents, then they were collected and lysed with RIPA buffer solution (Beyotime Institute of Biotechnology). The protein concentration was determined by BCA method (Beyotime Institute of Biotechnology). Samples $(25 \mu \mathrm{g})$ were separated via SDS-PAGE (10 or $12 \%$ gel) and transferred onto a polyvinylidene fluoride (PVDF) membrane (EMD Millipore). After blocking with 5\% Bovine serum albumin for $1 \mathrm{~h}$ at room temperature, the membranes were incubated with primary antibodies, including HMGB1, RAGE, p62, LC3II/I, Parp, P53, phospho-P53, bcl2, bax, Puma,cleaved caspase 3, erk, phospho-erk, NFkB/p65, phospho-NF-kB/p65, MRP, P-gp overnight at $4^{\circ} \mathrm{C}$. Secondary antibodies, including sheep antimouse IgG-HRP and sheep anti-rabbit IgG-HRP were applied at a 1:5,000 dilution for $1 \mathrm{~h}$ at room temperature. $\beta$-actin and GAPDH were used as loading controls to detect the expression in whole protein. The target protein expressions were detected with an enhanced chemiluminescence reagent (EMD Millipore) using a G:BOX XT4 system (Syngene).

\section{HMGB1 Elisa kit}

The HMGB1 Elisa kit was purchased from Elabscience Biotechnology Co,Ltd. Both Jurkat and HL-60 cells are treated with $\operatorname{ADM}(0,0.05,0.1,0.2,0.4,0.8 \mu \mathrm{M})$ for $24 \mathrm{~h}$, then the supernate was collected. We diluted the supernate at 1:2 and added into the wells. The supernate and the reaction mixture were incubated in a $37^{\circ} \mathrm{C}$ incubator for 30 minutes. Then the absorbance at $450 \mathrm{~nm}$ was measured in a microplate reader and we calculated each wells' content of HMGB1 according to the standard curve.

\section{Lentivirus infection}

To mutate the acetylation site of HMGB1, Jurkat cells that had been tranfected with shRNA-mutated acetylation site of HMGB1 was purchased and from OBiO Technology Corp., Ltd and was verified. To knock down the expression of RAGE, we chose three potential sequences, and lentiviruses were purchased from HanBio Technology (Shanghai, China). Experiments were performed in 12-well plates at

a density of $3 \times 10^{5}$ cells/well. In the present study, both Jurkat and HL-60 cells were divided into three groups, which consisted of the wild type, cells transformed with lentivirus that included Normal control and shRNA. 2ug/mL puromycin (Beijing Solarbio Science \& Technology Co., Ltd.) was applied to select cells that had been well transfected. Then we used qPCR and western blot to measure the expression of RAGE. Finally,we selected the best one that targeting RAGE and the shRNA sequence was as follow: forward,5'-GCCGGAAAUUGUGAAUCCUTT -3' and reverse, 5'-AGGUUCACAAUUUCCGGCTT -3'. Cells were cultured at $37^{\circ} \mathrm{C}$ in a humidified incubator containing $5 \% \mathrm{CO} 2$ for 3-5 generations and then used for subsequent experiments.

\section{Detection of autophagic flux.}


The mRFP-GFP-LC3 adenoviral particles were purchased from HanBio (Shanghai, China). Cells were infected with adenoviral particles; after infection, the cells were pretreated with $\mathrm{rHMGB} 1(50 \mathrm{ng} / \mathrm{mL})$ for $24 \mathrm{~h}$ before chemical treatment or were distinctly cultured with $\operatorname{ADM}(0.4 \mu \mathrm{M})$ for another $24 \mathrm{~h}$. Then cells was fixed with $4 \%$ paraformaldehyde for $20 \mathrm{~min}$ and was washed with PBS twice. Imaging was performed on CarlZeiss LSM710 and analysis was performed using ZEN 2009.

\section{Transmission electron microscopy.}

The cells were fixed with $2 \%$ paraformaldehyde and $2.5 \%$ glutaraldehyde in $0.1 \mathrm{~mol} / \mathrm{L}$ phosphate buffer $\left(\mathrm{pH} \mathrm{7.4)}\right.$ at $4^{\circ} \mathrm{C}$ for $2 \mathrm{~h}$, followed by $1 \%$ OsO4. After dehydration and Epon-812:100\% acetone embedding at room temperature, thin sections $(50-80 \mathrm{~nm})$ were stained with uranyl acetate and lead citrate respectively at $4^{\circ} \mathrm{C}$ for $15 \mathrm{~min}$, and viewed under a HITACHI囚HT7700囚 election microscope (FEl; Thermo Fisher Scientific, Inc).

\section{Flow Cytometry}

Both Jurkat and HL-60 cells that transfected with shRNA-NC and shRNA-RAGE were pre-treatd with or without extracelluar HMGB1 $(50 \mathrm{ng} / \mathrm{mL}$ ) for $24 \mathrm{~h}$, then $0.8 \mathrm{uM} \mathrm{ADM}$ was added for another $24 \mathrm{~h}$. Then leukemia cells were were washed with PBS for 2 times and then stained with Annexin V APC-A (ThermoFisher Scientific, US) and APC-Cy7-A (ThermoFisher Scientific) before its detection by flow cytometry (FACSCalibur, BD Biosciences, San Diego, CA).

\section{Animal study}

Jurkat cells $\left(5 \times 10^{6} / \mathrm{mL}\right)$ that stably transfected with shRNA-NC, shRNA-RAGE as well as shRNA-mutated acetylated site of HMGB1 was injected into NOD/SCID mouse via tail intravenous injection. There are 7 mice in each group. Low dosage of ADM $(1 \mathrm{mg} / \mathrm{kg})$ was injected into mouse by intraperitoneal injection every other day. $25 \%$ weight loss or live for 4 weeks were set as the end point. Then the spleen was removed to make pathological section by using hematoxylin-eosin staining. Futhermore, Bone marrow sediment of mouse was removed and stained with BV421-CD3 before flow cytometry to detect the proportion of leukemia cells in bone marrow. Part of them was subjected to Transmission electron microscopy to detect the level of autophagy. This study was approved by the Ethics Committee of the Sun Yat-Sen Memorial Hospital of Sun Yat-Sen University.

All data are expressed as the mean \pm standard deviation. Paired Student's t-test was used for comparisons between two groups and one-way analysis of variance (ANOVA) was performed for comparisons between more than two groups. $\mathrm{P}<0.05$ was considered to indicate a statistically significant result. All statistical analyses were conducted by SPSS version 18.0 software (SPSS, Inc.).

\section{Results}

Chemotherapeutic drug ADM could induce autophagy as well as apoptosis in leukemia cells. 
Adriamycin(ADM), also known as Doxorubicin, belongs to the family of anthracycline anticancer drugs, is the cornerstone of the chemotherapy of childhood acute leukemia(AL). As shown in Figure. 1A, both Jurkat and HL-60 cells were damaged by ADM in a dose-dependent manner. Western blotting also applied to analyze ADM's function on leukemia cells. Fig.1B depicted the upregulation of autophagy in both leukemia cells via western blotting analysis, reflected in the increased ratio of LC3II/I and the continuous decrease of P62, one of the substrate of autophagy. It can be seen in Fig.1C that both green and red puncta of LC3 were increased in ADM-treated group, which implied that the autophagy flux was augmented as the appliance of ADM in leukemia cells. What's more, we analyzed the changed of apoptosis when cells treated with ADM by western blotting. It was shown that the level of PARP, one of the substrate and initiate of apoptosis, as well as the ratio of Bcl 2/Bax were gradually decreased. In the meantime, the level of cleaved caspase 3 was increased, which indicated that apoptosis level was elevated when two cell lines treated with ADM(Fig. 1D). Above all, Fig. 1 demonstrates that autophagy and apoptosis are provoked by ADM in the leukemia cells.

\section{HMGB1 was upregulated and released when leukemia cells treated with ADM.}

As a damage-associated molecular pattern (DAMP) molecule, HMGB1 have been stated that its expression was upregulated in various kinds of cancer. In an attempt to characterize the role HMGB1 plays in acute leukemia, western blotting and HMGB1 Elisa kit were employed to analyze the expression of HMGB1. As presented in Fig.1B, the expression of HMGB1 was slightly upregulated in lower dose of $A D M$, however, in higher dose of ADM,the expression of HMGB1 was decreased. The result of Elisa illuminated that HMGB1 was released in a dose-dependent manner (Fig.1E). To sum up, HMGB1 was upregulated and released under the treatment of ADM, while what will the HMGB1 do when it is release to out of cell still require further research.

\section{Extracelluar HMGB1 promoted tumor cells survival by enhancing autophagy and diminishing apoptosis.}

To figure out extracelluar HMGB1's function on leukemia cells, we incubated the cells with extracelluar HMGB1 for 24h before treated with ADM. The changes of autophaghy and apoptosis were analyzed by western blotting. On one hand, it can be seen from Fig. 2 that P62 was continuously decreased and the ratio of LC3II/I was increased, which indicated that leukemia cells pretreated with rHMGB1 had a higher level of autophagy than those untreated with rHMGB1. On the other hand, apoptosis was restricted when pretreated with rHMGB1, which was embodied in the descending expression of cleaved caspase 3 as well as the ascending ratio of $\mathrm{Bcl} 2 / \mathrm{Bax}$ and the increase of total Parp. To sum up, extracelluar HMGB1 do protect cells from chemotherapeutic drug, which results from its regulations on autophagy and apoptosis in leukemia cells.

\section{Inhibited the expression of RAGE prevented autophagy induced by extracelluar HMGB1.}

To characterize the role RAGE plays in leukemia, we inhibited the expression of RAGE in two leukemia cell lines by lentivirus infection. Western blotting was used to analyze the expression of RAGE (Fig. 3A a-1). As shown in Figure. 3A a-4, leukemia cells were damaged by ADM while RAGE' cells were even worse and 
could not be protected by rHMGB1. Then experiments had been conducted to examined RAGE's role in HMGB1 induced autophagy. Detection of LC3II/I by immunoblotting or immunofluorescence delineated that knock down of RAGE curbed the autophagy and prevent the autophagy induced by HMGB1 (Fig. 3A a-2 and Fig. 3D). The limited degradation of P62 also showed that autophagy was prevented (Fig. 3A a2). There was a significant difference between NC and RAGE- group, which demonstrated that RAGE is crucial in the progression of autophagy.

\section{HMGB1/RAGE induced autopaghy was associated with increased level of phosphorylated ERK2/1 and decreased level of phosphorylated mTOR.}

Autophagy is initiated by many mechanism, in which PI3K/AKT/mTOR and MEK/ERK2/1 are crucial and frequently studied. To gain insight in the molecular mechanism of HMGB1/RAGE induced autophagy, we compared the difference of the level of phosphorylated ERK and phosphorylated mTOR between shRNANC and shRNA-RAGE cells. From Fig. 3A a-2, we can see that the level of phosphorylated ERK was declined while the level of phosphorylated mTOR was increased. To further research the relationship between erk, mTOR and autophagy, we used small molecule inhibitor of ERK (PD98059) and mTOR (Rapamycin) to curb the activation of ERK and mTOR. It can be seen from Fig. 3B that the level of phosphorylation of ERK was decreased autophagy was limited accordingly, which was ascertained by decreasing P62 and increasing ratio of LC3II/I in both RAGE knocked down tumor cells. Unexpectedly, phosphorylated mTOR was declined. When the shRNA-RAGE cells treated with Rapamycin, autophagy was upregulated as the phosphorylated mTOR declined. (Fig.3C c-1) According to the mentioned above, It is suggested that knocking down of RAGE limits autophagy induced by chemotherapy treatment, and the changes of the phosphorylation of ERK and mTOR were indispensable in this process.

\section{Inhibited the expression of RAGE increased apoptosis in leukemia cells and the apoptosis was related with the phosphorylated of P53.}

Apoptosis, also known as programmed cell death, is one of the best characterized form of cell-death process. As mentioned above, apoptosis was halted when employed extracelluar HMGB1 into leukemia cells. To identify the function of RAGE in the progression of apoptosis, firstly we knocked down RAGE and we can clearly see that the ascending cleaved caspase3, which indicates the upregulation of apoptosis in leukemia cells following chemical treatment. What's more, extracelluar HMGB1 had less protective impact or even become harmful on cells without RAGE (Fig. 4A and 4C). Furthermore, we found that depletion of RAGE increased phosphorylation of p53(Fig. 4A). P53 is the one of tumor supressor and is a major checkpoint. Activating P53 can initiate apoptosis when cells encounter intracellualar genotoxic stress. We also found that the level of pro-apototic bcl2-family members, Bax and PUMA in RAGE knocked down leukemia cells was higher than control group, while the level of anti-apoptosis protein $\mathrm{Bcl} 2$ was reduced. To clarify the role that P53 plays in apoptosis results from knocking down of RAGE. we use pififithrin $\beta$ (PFT- $\beta$ ), a specific antagonist of p53, to inhibit the phosphorylation of p53 in RAGE' leukemia cells. It can be seen from Fig. 4B that inactivate P53 increased the ratio of $\mathrm{Bcl} 2 / \mathrm{Bax}$ while downregulate the expression of PUMA following chemotherapy treatment. What's more, the phosphorylation of mTOR also 
decreased. Therefore, it is suggested that RAGE could limit apoptosis and this process was associated with the phosphorylation of P53.

\section{HMGB1/RAGE induced the expression of drug resistance protein by activating NF-KB pathway.}

Over-expression of drug resistance protein such as P-gp and MRP is one of the reasons of existence of multi-drug resistance (MDR) and obstacles of anti-cancer treatments. From Fig. 5A we can see that extracelluar HMGB1 actually could upregulate the expression of drug resistance protein, and this process was also RAGE-dependent. In the meantime the activation of NF-KB was halted due to the depletion of RAGE. In the subsequent experiment, PDTC, one of the antagonist of NF-KB , was applied before chemtherapy. Western blotting and qPCR was used to assess the expression of P-gp and MRP. Fig. 5B showed that the expression of P-gp and MRP was sharply decreased as the inactivation of NF-KB . It is implied that HMGB1/RAGE can provoke the expression of P-gp and MRP primarily by the activation of NF-KB .

\section{Blocking HMGB1/RAGE axis increased sensitivity to chemotherapy in vivo}

To figure out whether knockdown of RAGE increased sensitivity towards chemotherapy in vivo, we established a model of T-ALL mouse. Fig. 6A depicted that mouse injected with shRNA-RAGE or shRNAmutated acetylation site had a better life span than those injected with shRNA-NC cells. From Fig. 6B and $6 \mathrm{C}$ we can see that mouse that injected with shRNA-RAGE or shRNA-mutated acetylation site had less burden of leukemia cells in spleen and marrow. As shown in Fig.6D, transmission electron microscopy was employed to calculate the number of autophagosome-like structures and it was suggested that mouse that injected with shRNA-RAGE or shRNA-mutated acetylation site had a lower level of autophagy than those with shRNA-NC cells. From above mentioned, it is suggested that the interference of HMGB1/RAGE can alleviate the leukemia burden in vivo.

\section{Discussion}

Currently, drug resistance remains the main obstacle to overcome when treating acute leukemia with chemotherapy treatment, which is also the primary reason for the development of refractory and recurrent leukemia ${ }^{[6,41]}$. A great number of studies had focused on this aspect, in which the elevation of autophagy and the over-expression of multiple drug-resistant protein as well as the suppression of apoptosis were demonstrated to contribute greatly to the occurrence of drug resistance. ADM is one of the cornerstone chemotherapy drugs for leukemia, and the cytotoxicity mediated by ADM is drug-induced DNA damage ${ }^{[41]}$. High-mobility group protein $1(\mathrm{HMGB} 1)$, known as one of the most characterized damage associated molecular pattern molecule(DAMP), could be passively released when cells are under stress or injured, leading to the regulation of autophagy, apoptosis as well as immunity and so on ${ }^{[42]}$. Han et al demonstrated that ADM could trigger the translocation and release of HMGB1 in tumor cells, which induced cytoprotective autophagy in K562 cells by activating the MEK/ERK2/1 signalling pathway ${ }^{[43]}$. Kong et al revealed that HMGB1 promoted the dissociation of Beclin1-Bcl-2 complexes and modified 
Beclin 1 binding to PI3k catalytic subunit 3, thus initiating autophagosome formation ${ }^{[25]}$. Liu et al showed that extracelluar HMGB1 can promote autophagy and prevent necroptosis in acute myeloid leukemia cells $^{[17]}$. In this study, as shown in Fig. 1, ADM do upregulate autophagy and apoptosis in a dosedependent manner accompanied by the release of HMGB1 in acute leukemia cell, which was in accordance with the studies mentioned above. However, the overall expression of HMGB1 was not significantly changed, at the higher dosage of ADM, the expression of HMGB1 was sharply decreased. Combined with the result of Elisa, we assumed that leukemia cells might encounter necroptosis and HMGB1 was released rapidly. As presented in Fig. 2, we pretreated leukemia cells with rHMGB1 before treated with ADM and found that extracelluar promoted autophagy and protect cells from apoptosis, which suggested that extracelluar HMGB1 play an protective role in chemotherapeutic treatment. However, how the released HMGB1 acts on leukemia cells remain elusive.

The receptor for advanced glycation end products(RAGE) is the first demonstrated binding partner to $H M G B 1^{[44]}$. RAGE is a multiligand receptor of the immunoglobulin superfamily of cell surface molecules acting as counter-receptor for diverse molecules such as AGEs, S100 proteins, HMGB1 and so on ${ }^{[29]}$. RAGE and its ligands are over-expressed in multiple cancers ${ }^{[34,45]}$. What's more, it was reported that HMGB1-RAGE signalling triggered activation of key cell signalling pathways, such as MAPK, NF-KB, and $\mathrm{Rac} / \mathrm{Cdc} 42$ in nasopharyngeal carcinoma ${ }^{[46]}$. Study had also found that in a murine model, targeted knocking down of RAGE diminished the autophagy and prevented the development of early pancreatic neoplasia possibly through IL-6-induced phosphorylation of STAT3 ${ }^{[47]}$. In pancreatic cancer, it was reported that HMGB1 or its receptor RAGE by RNAi or antisense nucleotide could inhibit cell invasion and augment chemotherapy sensitivity partly by down-regulation of autophagy. ${ }^{[48]}$ Therefore, we hypothesized that extracelluar HMGB1 acts on leukemia cells mainly by interacting with RAGE. In present study, by targeting knocking down RAGE, we found that the level of autophagy was sharply decreased and the apoptosis was increased in both leukemia cell lines(Fig. 3). What's more, the absence of RAGE halted the protection of rHMGB1. Leukemia cells was aggravated even worse with HMGB1 due to the ablation of RAGE. On one hand, studies found that extracelluar HMGB1 can interact with other receptors such as TLR4 and CXCR4 and then worsen inflammation. On the other hand, the arsing ROS level can oxidize HMGB1, which makes it more liable to bind to TLR4 ${ }^{[24,27]}$, and then leads to the deterioration of leukemia cells.

As shown in Fig.7, MAPK/ERK and mTOR are crucial in the initial of autophagy. Pan et al recently reported that HMGB1 enhanced autophagy through activating the MEK/ERK1/2 signalling pathway and promote docetaxel resisitance in human lung adenocarcinoma ${ }^{[49]}$. Li et al postulated that HMGB1 facilitates the growth and advancement of clear cell renal cell carcinoma via ERK1/2 stimulation, which is partly mediated by RAGE ${ }^{[50]}$. While Liu et al reported that HMGB1 enhanced autophagy and chemotherapy resistance through MAPK/mTOR signaling pathway in $\mathrm{K} 562$ cells $^{[51]}$. In present study, we make an attempt to figure out the relationship between ERK and mTOR in autophagy induced by HMGB1/RAGE. We found that in RAGE' cells,the phosphorylation of ERK was decrease and by contrast, 
the phosphorylation of mTOR was elevated, along with the restriction of autophagy. By inhibiting the phosphorylation of ERK by PD98059, a small molecular inhibitor of MEK/ERK, strikingly decreased autophagy in leukemia cells. To our surprise, the phosphorylation of mTOR was repressed. Then Rapamycin was applied to the RAGE- cells, autophagy was upregulated conversly as the phosphorylation of $\mathrm{mTOR}$ was reduced.

It was believed that Beclin-1 is crucial in the autophagy initiated by the activation of mTOR, while the dislocation of Beclin-1 and Bcl 2 resulted from the activation of MEK/ERK. And it is suggested that the absence of RAGE lead to down-regulation of autophagy via the increased phosphorylation of mTOR and the decreased phosphorylation of ERK, in which MEK/ERK suppose to be the upstream of the mTOR signal. The activation of ERK is more crucial in the autophagy induced by HMGB1/RAGE.

P53, one of the most studied tumor suppressor gene, had been found frequently mutated in leukemia cells and assumed to have a cross link to HMGB1 to regulate autophaghy and apoptosis in the setting of cell stress ${ }^{[52]}$. What's more, P53 induced apoptosis, known as intrinsic apoptosis, is mainly by activating the expression of pro-apoptotic protein such as PUMA and Bax, along with the decline of anti-apoptotic protein such as Bcl-2 and $\mathrm{MCl}-1^{[13,53]}$. HMGB1/RAGE also have great association with apoptosis. It is found that HMGB1-mediated autophagy decreases vincristine-induced apoptosis in gastric cancer partly via upregulation of $\mathrm{Mcl}-1$, a Bcl-2 family member ${ }^{[54]}$. In present study, We found that targeted knocking down RAGE induce apoptosis and prevented the protective effect of extracelluar HMGB1 with the change of the Bcl-2 family member. We proposed that HMGB1/RAGE related apoposis is P53 dependent. The increased phosphorylation of P53 was observed in RAGE- cells. And then we use pifithrin B , a small molecular inhibitor of P53 to inhibit the activation of P53 and we found that it abrogated the enhanced apoptosis due to the ablation of RAGE. Meanwhile, we can see that the decline of PUMA and Bax and the increase of Bcl-2 when treated with pifithrin B(Fig. 4). To our interest, the decrease the level of phosphorylation of mTOR was reduced, which suggested that P53 can be the hub of the regulation of autophagy and apoptosis related with HMGB1/RAGE. The association between P53 and HMGB/RAGE require further research.

It is established that NF-KB is one of the signaling way activated by HMGB1/RAGE ${ }^{[16,55,56]}$ And NF-KB is a key protein that affect the progression of tumor. Upon activation, NF-KB translocates to the nucleus and subsequently binds to DNA sequences to stimulate transcription of target genes, $A B C$ family members such as $A B C C 1$ and $A B C B 1$ were included ${ }^{[57-59]}$. $A B C C 1$ and $A B C B 1$ transporters conferred resistance to multidrug resistance (MDR), an intrinsic or acquired cross-resistance toward different chemotherapeutic drugs, which has been considered as one of the primary reasons for the failure in cancer chemotherapy. Meanwhile, numberous studies demonstrated that anthracyclines such as doxorubicin and daunorubicin were substrates of MRP1 and P-gp, encoded by $A B C C 1$ and $A B C B 1^{[35,60]}$. However, the relationship between HMGB1/RAGE and ABC transporters are unclear. In present study, the phosphorylation of NF-KB as well as the expression of P-gp and MRP was decreased followinng the absence of HMGB1 and RAGE. When used a small molecule inhibitor PDTC to prevent the phosphorylation of NF-KB, the expression of P- 
gp and MRP were decreased both in mRNA and protein level . It indicated that HMGB1/RAGE might induce NF-KB to translocate into nucleus and promoting the expression of $A B C B 1$ and $A B C C 1$.

Acetylation of HMGB1 is crucial in the translocation and release of $\mathrm{HMGB1}^{[23]}$. Mice that injected with mutated HMGB1 cells or RAGE- cells had better life quality and less leukimia burden. What's more, the result of transmission microscope validated the important role HMGB1/RAGE play in autophagy. It is suggested that HMGB1, as a DAMP molecule, was upregulated and release to adapt leukemia cells to chemotherapy, while RAGE, acting as HMGB1's receptor, affect downstream signaling and promote leukemia cell survival by regulations of autophagy, apoptosis as well as multidrug resistant proteins. To sum up, HMGB1/RAGE shape the accommodating micro-environment for leukemia cells to prevent the harm of chemotherapy.

In conclusion, the present study demonstrate the role HMGB1/RAGE axis plays in promoting acute leukemia cells survival by diminishing apoptosis and increasing autophagy and the expression of multiple drug resistant protein. These data support the development of novel therapy targeting HMGB1/RAGE. Interfering with HMGB1/RAGE can be a potential means to cure acute leukemia.

\section{Declarations}

\section{Acknowledgements}

\section{Funding}

This study was supported by National Natural Science Foundation of China (grant no. 81100370) and Guangzhou science and technology plan projects (grant no. 201803010032).

\section{Availability of data and materials}

The datasets used and/or analysed during the present are available from the corresponding author on reasonable request.

\section{Authors' contributions}

WEIXIN LAI and JIANPEI FANG designed the study. WEIXIN LAI and HAN CHEN collated and analyzed the data. WEIXIN LAI wrote the manuscript. QIAN KONG and XINYU LI participated in revising manuscript. YUNYAO LI collected background information. All authors have read and approved the final submitted manuscript.

\section{Ethics approval}

\section{Consent for publication}


All authors involved in the authorship are consent for publication in the current form.

\section{Competing interests}

The authors declare that they have no competing interests.

\section{Abbreviations}

\begin{tabular}{ll} 
HMGB1 & High mobility group box 1 \\
\hline ADM & Adriamycin \\
\hline RAGE & receptor for advanced glycation end products \\
\hline DAMP & damage-associated molecular pattern \\
\hline MAPK & mitogen activated protein kinases \\
\hline ERK & extra cellular signal regulated kinases \\
\hline AMPK & (AMP)-activated protein kinase \\
\hline P-gp & P-glycoprotein \\
\hline MRP & ATP-binding cassette transporter \\
\hline ABC & ATP-binding cassette transporter \\
\hline NF-KB & Nuclear factor KB \\
\hline LC3 & Microtubule-associated protein 1 light chain 3 \\
\hline P62 & Sequestosome 1 \\
\hline PI3K & Phosphatidylinositol-3-Kinase \\
\hline mTOR & Mammalian Target of Rapamycin
\end{tabular}

\section{References}

[1] Seth R, Singh A. Leukemias in Children[J]. The Indian Journal of Pediatrics, 2015,82(9):817-824.

[2] Karrman K, Johansson B. Pediatric T-cell acute lymphoblastic leukemia[J]. Genes, Chromosomes and Cancer, 2017,56(2):89-116.

[3] Hoffman A E, Schoonmade L J, Kaspers G J. Pediatric relapsed acute myeloid leukemia: a systematic review[J]. Expert Rev Anticancer Ther, 2021,21(1):45-52.

[4] Shen S, Cai J, Chen J, et al. Long-term results of the risk-stratified treatment of childhood acute lymphoblastic leukemia in China[J]. Hematological Oncology, 2018,36(4):679-688. 
[5] Hunger S P, Lu X, Devidas M, et al. Improved Survival for Children and Adolescents With Acute Lymphoblastic Leukemia Between 1990 and 2005: A Report From the Children's Oncology Group[J]. Journal of Clinical Oncology, 2012,30(14):1663-1669.

[6] Sarmento-Ribeiro A B, Scorilas A, Gonçalves A C, et al. The emergence of drug resistance to targeted cancer therapies: Clinical evidence[J]. Drug Resistance Updates, 2019,47:100646.

[7] Sehgal A R, Konig H, Johnson D E, et al. You eat what you are: autophagy inhibition as a therapeutic strategy in leukemia[J]. Leukemia, 2015,29(3):517-525.

[8] Sever O N, Demir O G. Autophagy: Cell death or survive mechanism[J]. Journal of oncological science, 2017,3(2):37-44.

[9] Schuler D, Szende B. Apoptosis in acute leukemia[J]. Leukemia Research, 2004,28(7):661-666.

[10] Hanahan D, Weinberg R A. Hallmarks of Cancer: The Next Generation[J]. Cell, 2011,144(5):646-674.

[11] Evangelisti C, Evangelisti C, Chiarini F, et al. Autophagy in acute leukemias: A double-edged sword with important therapeutic implications[J]. Biochimica et Biophysica Acta (BBA) - Molecular Cell Research, 2015,1853(1):14-26.

[12] Nikoletopoulou V, Markaki M, Palikaras K, et al. Crosstalk between apoptosis, necrosis and autophagy[J]. Biochimica et Biophysica Acta (BBA) - Molecular Cell Research, 2013,1833(12):3448-3459.

[13] Xu J, Patel N H, Gewirtz D A. Triangular Relationship between p53, Autophagy, and Chemotherapy Resistance[J]. International Journal of Molecular Sciences, 2020,21(23):8991.

[14] Yang L, Yu Y, Kang R, et al. Up-regulated autophagy by endogenous high mobility group box-1 promotes chemoresistance in leukemia cells[J]. Leuk Lymphoma, 2012,53(2):315-322.

[15] Zhao M, Yang M, Yang L, et al. HMGB1 regulates autophagy through increasing transcriptional activities of JNK and ERK in human myeloid leukemia cells[J]. BMB Reports, 2011,44(9):601-606.

[16] Huang Z, Zhong Z, Zhang L, et al. Down-regulation of HMGB1 expression by shRNA constructs inhibits the bioactivity of urothelial carcinoma cell lines via the NF-KB pathway[J]. Scientific Reports, 2015,5(1).

[17] Liu Y, Chen P, Xu L, et al. Extracellular HMGB1 prevents necroptosis in acute myeloid leukemia cells[J]. Biomedicine \& pharmacotherapy, 2019,112:108714.

[18] Huang C, Chiang S, Chen W T, et al. HMGB1 promotes ERK-mediated mitochondrial Drp1 phosphorylation for chemoresistance through RAGE in colorectal cancer[J]. Cell Death \& Disease, 2018,9(10). 
[19] Yin H, Yang X, Gu W, et al. HMGB1-mediated autophagy attenuates gemcitabine-induced apoptosis in bladder cancer cells involving JNK and ERK activation[J]. Oncotarget, 2017,8(42):71642-71656.

[20] Yuan S, Liu Z, Xu Z, et al. High mobility group box 1 (HMGB1): a pivotal regulator of hematopoietic malignancies[J]. Journal of hematology and oncology, 2020,13(1):1-91.

[21] A. Richard S. High-mobility group box 1 is a promising diagnostic and therapeutic monitoring biomarker in Cancers: A review[J]. 2018,5(4):183-241.

[22] Farahat $M$, Sharaf $M$, Attia $T$. The serum high-mobility group box 1 level and RAGE expression in childhood acute lymphoblastic leukemic patients'[J]. The Egyptian Journal of Haematology, 2015,40(2):60.

[23] Li Y, Xie J, Li X, et al. Poly (ADP-ribosylation) of HMGB1 facilitates its acetylation and promotes HMGB1 translocation-associated chemotherapy-induced autophagy in leukaemia cells[J]. Oncol Lett, 2020,19(1):368-378.

[24] Kang R, Livesey K M, Zeh H R, et al. HMGB1 as an autophagy sensor in oxidative stress[J]. Autophagy, 2011,7(8):904-906.

[25] KONG Q, XU L, XU W, et al. HMGB1 translocation is involved in the transformation of autophagy complexes and promotes chemoresistance in leukaemia[J]. International Journal of Oncology, 2015,47(1):161-170.

[26] Andersson U, Yang H, Harris H. High-mobility group box 1 protein (HMGB1) operates as an alarmin outside as well as inside cells[J]. Seminars in Immunology, 2018,38:40-48.

[27] Tang D, Kang R, Cheh C W, et al. HMGB1 release and redox regulates autophagy and apoptosis in cancer cells[J]. Oncogene, 2010,29(38):5299-5310.

[28] Jangde N, Ray R, Rai V. RAGE and its ligands: from pathogenesis to therapeutics[J]. Critical reviews in biochemistry and molecular biology, 2020,55(6):555-575.

[29] Bucciarelli $L$ G, Wendt T, Rong $L$, et al. RAGE is a multiligand receptor of the immunoglobulin superfamily: implications for homeostasis and chronic disease[J]. Cell Mol Life Sci, 2002,59(7):11171128.

[30] El-Far A H, Sroga G, Al Jaouni S K, et al. Role and Mechanisms of RAGE-Ligand Complexes and RAGE-Inhibitors in Cancer Progression[J]. International Journal of Molecular Sciences, 2020,21(10):3613.

[31] Kang R, Tang D, Livesey K M, et al. The Receptor for Advanced Glycation End-Products (RAGE) Protects Pancreatic Tumor Cells Against Oxidative Injury[J]. Antioxidants \& Redox Signaling, 2011,15(8):2175-2184. 
[32] Fuentes M K, Nigavekar S S, Arumugam T, et al. RAGE Activation by S100P in Colon Cancer Stimulates Growth, Migration, and Cell Signaling Pathways[J]. Diseases of the Colon \& Rectum, 2007,50(8):1230-1240.

[33] Kang R, Tang D, Schapiro N E, et al. The receptor for advanced glycation end products (RAGE) sustains autophagy and limits apoptosis, promoting pancreatic tumor cell survival[J]. Cell Death Differ, 2010,17(4):666-676.

[34] Bierhaus A, Humpert P M, Morcos M, et al. Understanding RAGE, the receptor for advanced glycation end products[J]. Journal of Molecular Medicine, 2005,83(11):876-886.

[35] Fletcher $\mathrm{J} \mathrm{I,} \mathrm{Haber} \mathrm{M,} \mathrm{Henderson} \mathrm{M} \mathrm{J,} \mathrm{et} \mathrm{al.} \mathrm{ABC} \mathrm{transporters} \mathrm{in} \mathrm{cancer:} \mathrm{more} \mathrm{than} \mathrm{just} \mathrm{drug} \mathrm{efflux}$ pumps[J]. Nature Reviews Cancer, 2010,10(2):147-156.

[36] Fazlina N, Maha A, Zarina A L, et al. Assessment of P-gp and MRP1 activities using MultiDrugQuant Assay Kit: a preliminary study of correlation between protein expressions and its functional activities in newly diagnosed acute leukaemia patients[J]. Malaysian journal of pathology, 2008,30(2):87-93.

[37] Morrish E, Copeland A, Moujalled D M, et al. Clinical MDR1 inhibitors enhance Smac-mimetic bioavailability to kill murine LSCs and improve survival in AML models[J]. Blood Advances, 2020,4(20):5062-5077.

[38] Legrand O, Simonin G, Beauchamp-Nicoud A, et al. Simultaneous activity of MRP1 and Pgp is correlated with in vitro resistance to daunorubicin and with in vivo resistance in adult acute myeloid leukemia[J]. Blood, 1999,94(3):1046-1056.

[39] YIN Y, LI W, DENG M, et al. Extracellular high mobility group box chromosomal protein 1 promotes drug resistance by increasing the expression of P-glycoprotein expression in gastric adenocarcinoma cells[J]. Molecular Medicine Reports, 2014,9(4):1439-1443.

[40] Zhao B X, Sun Y B, Wang S Q, et al. Grape seed procyanidin reversal of p-glycoprotein associated multi-drug resistance via down-regulation of NF-kappaB and MAPK/ERK mediated YB-1 activity in A2780/T cells[J]. PLoS One, 2013,8(8):e71071.

[41] Chen C, Lu L, Yan S, et al. Autophagy and doxorubicin resistance in cancer[J]. Anti-Cancer Drugs, 2018,29(1):1-9.

[42] Muller S, Scaffidi P, Degryse B, et al. New EMBO members' review: the double life of HMGB1 chromatin protein: architectural factor and extracellular signal[J]. EMBO J, 2001,20(16):4337-4340.

[43] Han W, Sun J, Feng L, et al. Autophagy inhibition enhances daunorubicin-induced apoptosis in K562 cells[J]. PloS one, 2011,6(12):e28491. 
[44] Hori O, Brett J, Slattery T, et al. The Receptor for Advanced Glycation End Products (RAGE) Is a Cellular Binding Site for Amphoterin[J]. The Journal of biological chemistry, 1995,270(43):25752.

[45] Kalea A Z, See F, Harja E, et al. Alternatively Spliced RAGEv1 Inhibits Tumorigenesis through Suppression of JNK Signaling[J]. Cancer Research, 2010,70(13):5628-5638.

[46] Wu D, Ding Y, Wang S, et al. Increased expression of high mobility group box 1 (HMGB1) is associated with progression and poor prognosis in human nasopharyngeal carcinoma[J]. The Journal of Pathology, 2008,216(2):167-175.

[47] Kang R, Tang D, Lotze M T, et al. AGER/RAGE-mediated autophagy promotes pancreatic tumorigenesis and bioenergetics through the IL6-pSTAT3 pathway[J]. Autophagy, 2012,8(6):989-991.

[48] Kang R, Livesey K M, Zeh I H J, et al. Metabolic regulation by HMGB1-mediated autophagy and mitophagy[J]. Autophagy, 2011,7(10):1256-1258.

[49] Pan B, Chen D, Huang J, et al. HMGB1-mediated autophagy promotes docetaxel resistance in human lung adenocarcinoma[J]. Mol Cancer, 2014,13:165.

[50] Li J, Sun J, Rong R, et al. HMGB1 promotes myeloid-derived suppressor cells and renal cell carcinoma immune escape[J]. Oncotarget, 2017,8(38):63290-63298.

[51] Liu L, Gao F, Ye Y, et al. [Influence of HMGB1/MAPK/m-TOR signaling pathway on cell autophagy and chemotherapy resistance in K562 cells][J]. Zhong Nan Da Xue Xue Bao Yi Xue Ban, 2016,41(10):10161023.

[52] Livesey K M, Kang R, Vernon P, et al. p53/HMGB1 Complexes Regulate Autophagy and Apoptosis[J]. Cancer Research, 2012,72(8):1996-2005.

[53] Aubrey B J, Kelly G L, Janic A, et al. How does p53 induce apoptosis and how does this relate to p53mediated tumour suppression?[J]. Cell death and differentiation, 2018,25(1):104-113.

[54] Kang R, Chen R, Zhang Q, et al. HMGB1 in health and disease[J]. Mol Aspects Med, 2014,40:1-116.

[55] Kalea A Z, Reiniger N, Yang $\mathrm{H}$, et al. Alternative splicing of the murine receptor for advanced glycation end-products (RAGE) gene[J]. FASEB J, 2009,23(6):1766-1774.

[56] Xie J, Méndez J D, Méndez-Valenzuela V, et al. Cellular signalling of the receptor for advanced glycation end products (RAGE)[J]. Cellular signalling, 2013,25(11):2185-2197.

[57] YIN Y, LI W, DENG M, et al. Extracellular high mobility group box chromosomal protein 1 promotes drug resistance by increasing the expression of P-glycoprotein expression in gastric adenocarcinoma cells[J]. Molecular medicine reports, 2014,9(4):1439-1443. 
[58] Yu L, Li L, Medeiros L J, et al. NF-kB signaling pathway and its potential as a target for therapy in lymphoid neoplasms[J]. Blood Reviews, 2017,31(2):77-92.

[59] Karin M, Greten F R. NF-KB: linking inflammation and immunity to cancer development and progression[J]. Nature Reviews Immunology, 2005,5(10):749-759.

[60] Fukuda Y, Lian S, Schuetz J D. Leukemia and ABC transporters[J]. Advances in cancer research, 2015,125:171-196.

\section{Figures}



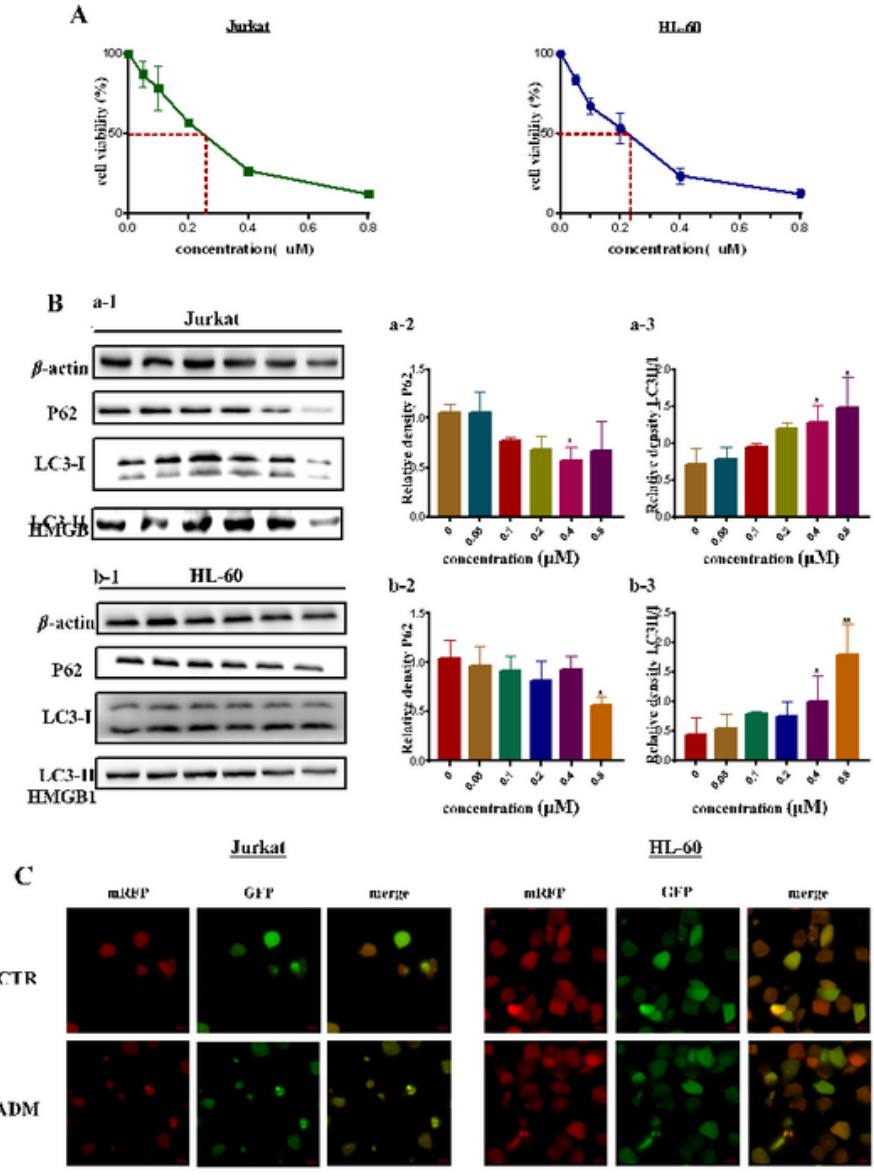

ADM
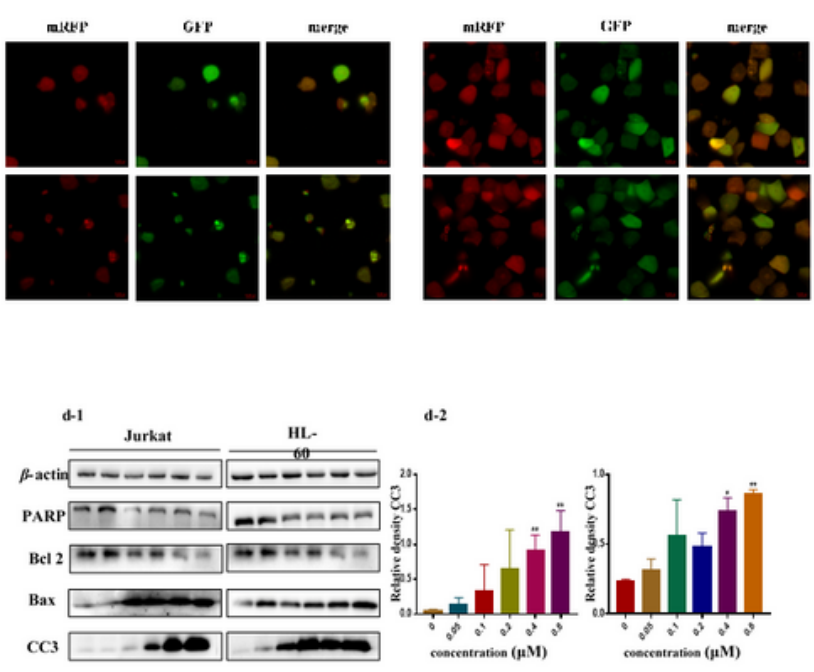

d-2

E

Jurkat

HI-60
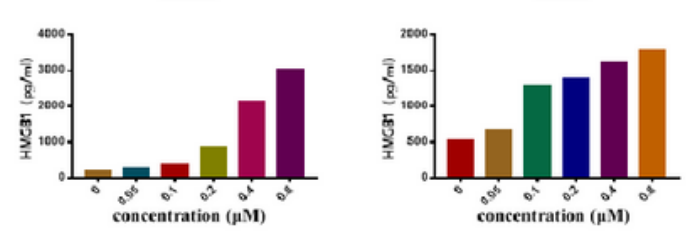

\section{Figure 1}

Chemotherapeutic drug ADM induced autophagy and apoptosis in acute leukemia cells. (A) ADM damaged both Jurkat and HL-60 cells dose-dependently. Cell Counting Kit-8 was used to assess the Cell viability. (B) Western blotting was used to detect the expression of LC3II/I and P62 in which $\beta$-actin was loading control. Both (a1-a2) and (b1-b2) diagrams are the quantitative data of LC3II/I and P62 in Jurkat and HL-60 cells. (C) Both Jurkat and HL-60 cells that stably expressed mRFP-EGFP-LC3 fusion protein 
were co-cultured with ADM for $24 \mathrm{~h}$. Confocal microscopic analysis is shown with $630 \times$ magnification. bar $=10 \mu \mathrm{m}(\mathrm{D})$ Western blotting was used to detect the expression of total PAPR, Bcl 2, Bax and cleaved caspase 3 to analyze the level of apoptosis. (d1-d2) were the diagrams of the quantitative data of cleaved caspase 3 in both leukemia cells. (E) HMGB1 was released when leukemia cells treated with ADM. HMGB1 Elisa kit was applied to assess the supernatant of different group. Data are the mean \pm standard deviation of three independent experiments. ${ }^{*} P<0.05,{ }^{*} \mathrm{P}<0.01$, compared with the untreated group. ADM, Adriamycin.
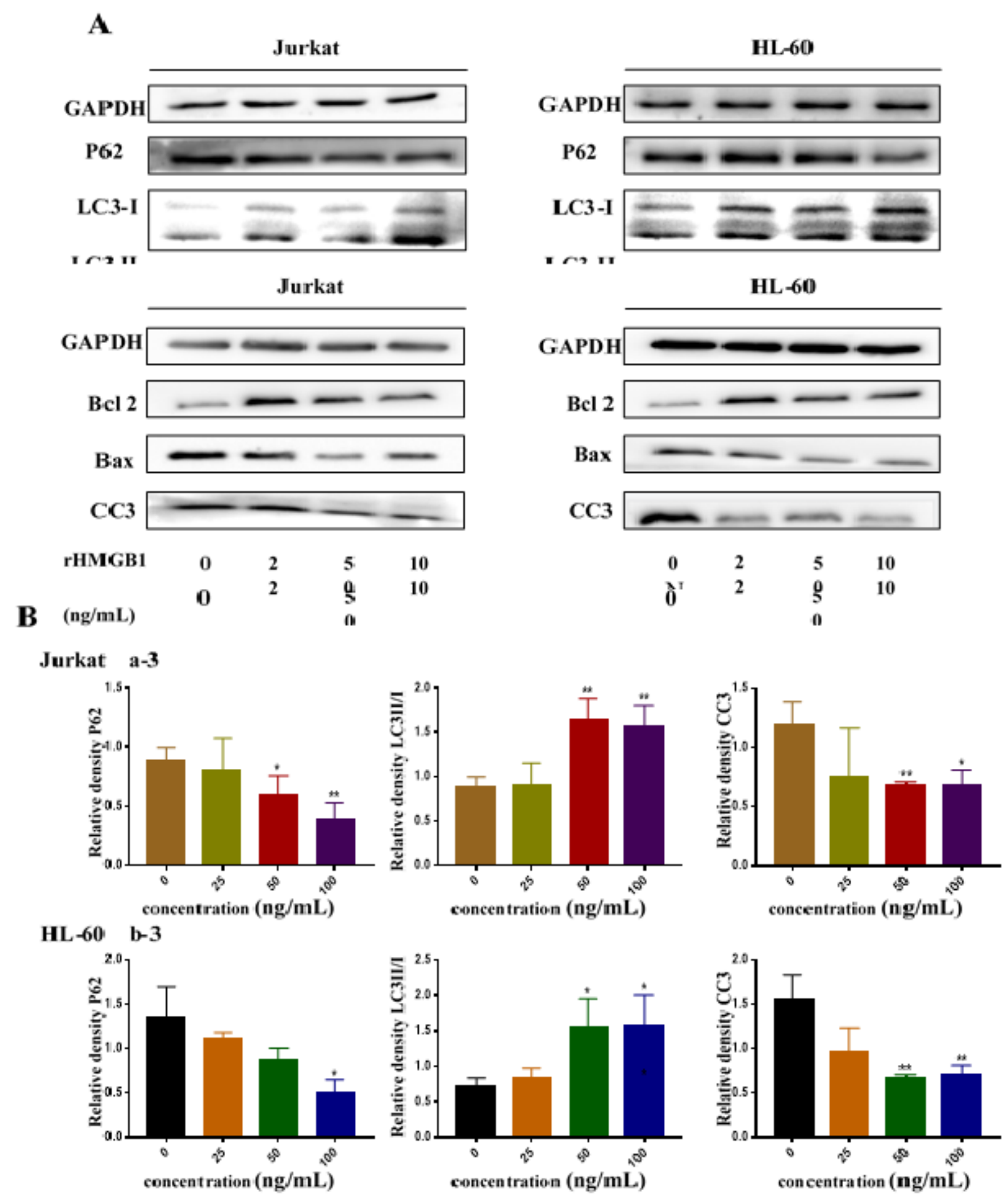

Figure 2 
Extracelluar HMGB1 induced autophagy and diminished apoptosis in acute leukemia cells. (A) Extracelluar HMGB1 protected leukemia cells from the damage of ADM by promoting autophagy and dimishing apoptosis, rHMGB1 $(0,25,50,100 \mathrm{ng} / \mathrm{mL})$ was added into leukemia cells before the appliance of $\operatorname{ADM}(0.4 \mu \mathrm{M})$,. Both (a-3) and (b-3) diagram were the quantitative data of LC3II/I and P62 as well as cleaved caspase 3 in Jurkat and HL-60 cells. GAPDH was used as loading control. Data are the mean \pm standard deviation of three independent experiments. ${ }^{*} P<0.05,{ }^{*} P<0.01,{ }^{*} * P<0.001$ compared with the untreated group.
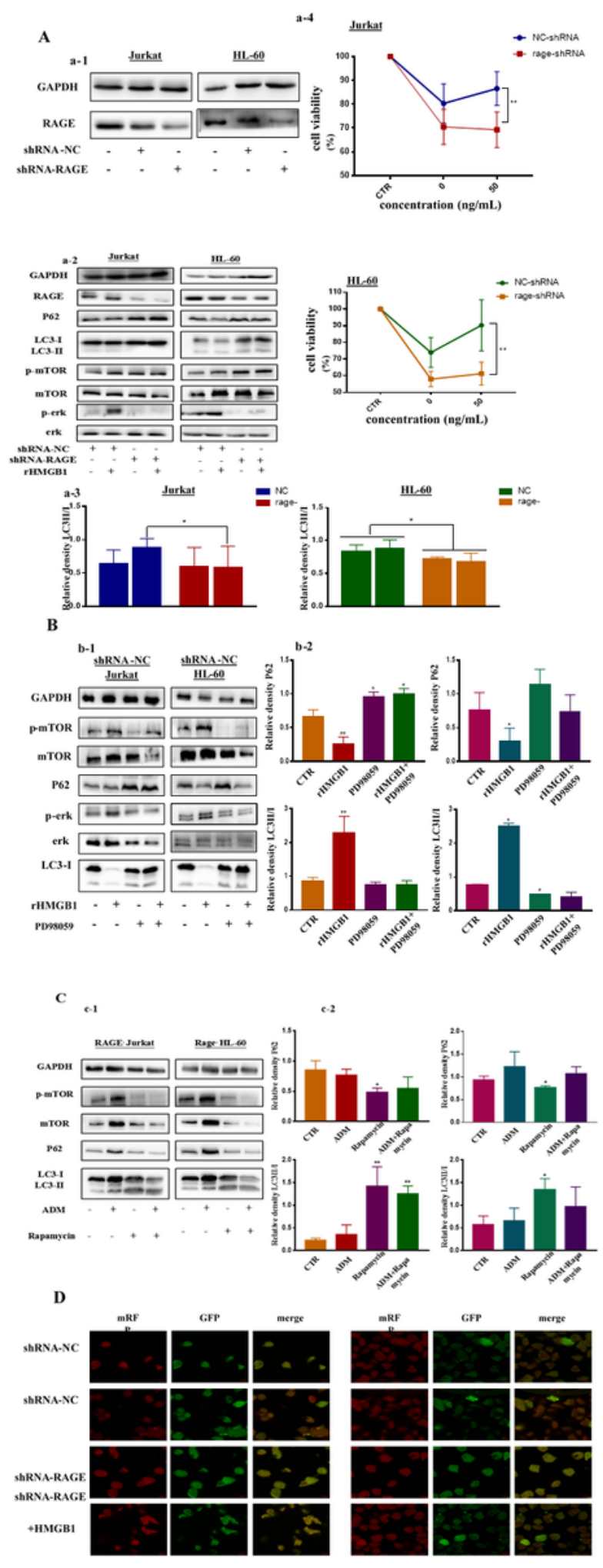


\section{Figure 3}

Targeted knocking down of RAGE restricted autophagy which was associated with the phosphorylation of erk and mTOR. (A) (a-1) Both Jurkat and HL-60 cells was transfected with shRNA-RAGE and shRNA-NC for 2 weeks. Then cells were subjected to western blotting to verify the knock down of RAGE protein. (a-2) Targeted knocking down of RAGE decreased autophagy that extracelluar HMGB1 induced in leukemia cells. Cells that stably transfected with shRNA-NC and shRNA-RAGE were pretreated with $50 \mathrm{ng} / \mathrm{mL}$ rHMGB1 for $24 \mathrm{~h}$, then cells were co-cultured with $0.4 \mu \mathrm{M}$ ADM for another $24 \mathrm{~h}$. Western blotting was used to detect the alternation of autophagy .(a-3) was the diagram of the quantitative data of LC3II/I in Jurkat and HL-60 cells respectively. .(a-4) Both leukemia cells were pretreated with or without rHMGB1 for 24h, then $\operatorname{ADM}(0.4 \mu \mathrm{M})$ was applied. Cell Counting Kit-8 was used to assess the Cell viability. (B) (b-1) Leukemia cells that tranfected with shRNA-NC were treated with a small inhibitor of ERK2/1, $10 \mu \mathrm{M}$ PD98059 together with $50 \mathrm{ng} / \mathrm{mL}$ rHMGB1 for $12 \mathrm{~h}$, then $0.4 \mu \mathrm{M}$ ADM was added into cells. Western blotting was used to detect the change of autophagy and the phosphorylation of ERK and mTOR. (b-2) showed the quantitative data of P62 and LC3II/I of leukemia cells. (C) Cells that stably transfected with shRNA-RAGE were pretreated with the small inhibitor of mTOR, Rapamycin(100nM) for $6 \mathrm{~h}$, following the treatment of $\operatorname{ADM}(0.4 \mu \mathrm{M})$. Cells were subjected to western blotting to assess the level of autophagy and the phosphorylation of mTOR. (c-2) presented the quantitative data of P62 and LC3II/I of leukemia cells. GAPDH was used as loading control. (D) Both Jurkat and HL-60 cells that transfected with shRNA-NC and shRNA-RAGE were tranfected with mRFP-EGFP-LC3 virus. In the susequent experiment, cells were pretreated with $50 \mathrm{ng} / \mathrm{mL}$ rHMGB1 for $24 \mathrm{~h}$ and then $0.4 \mu \mathrm{M}$ ADM was added for another $24 \mathrm{~h}$. Confocal microscopic analysis is shown with $630 \times$ magnification. Data are the mean \pm standard deviation of three independent experiments. ${ }^{*} P<0.05,{ }^{*} P<0.01$, compared with the untreated group. 

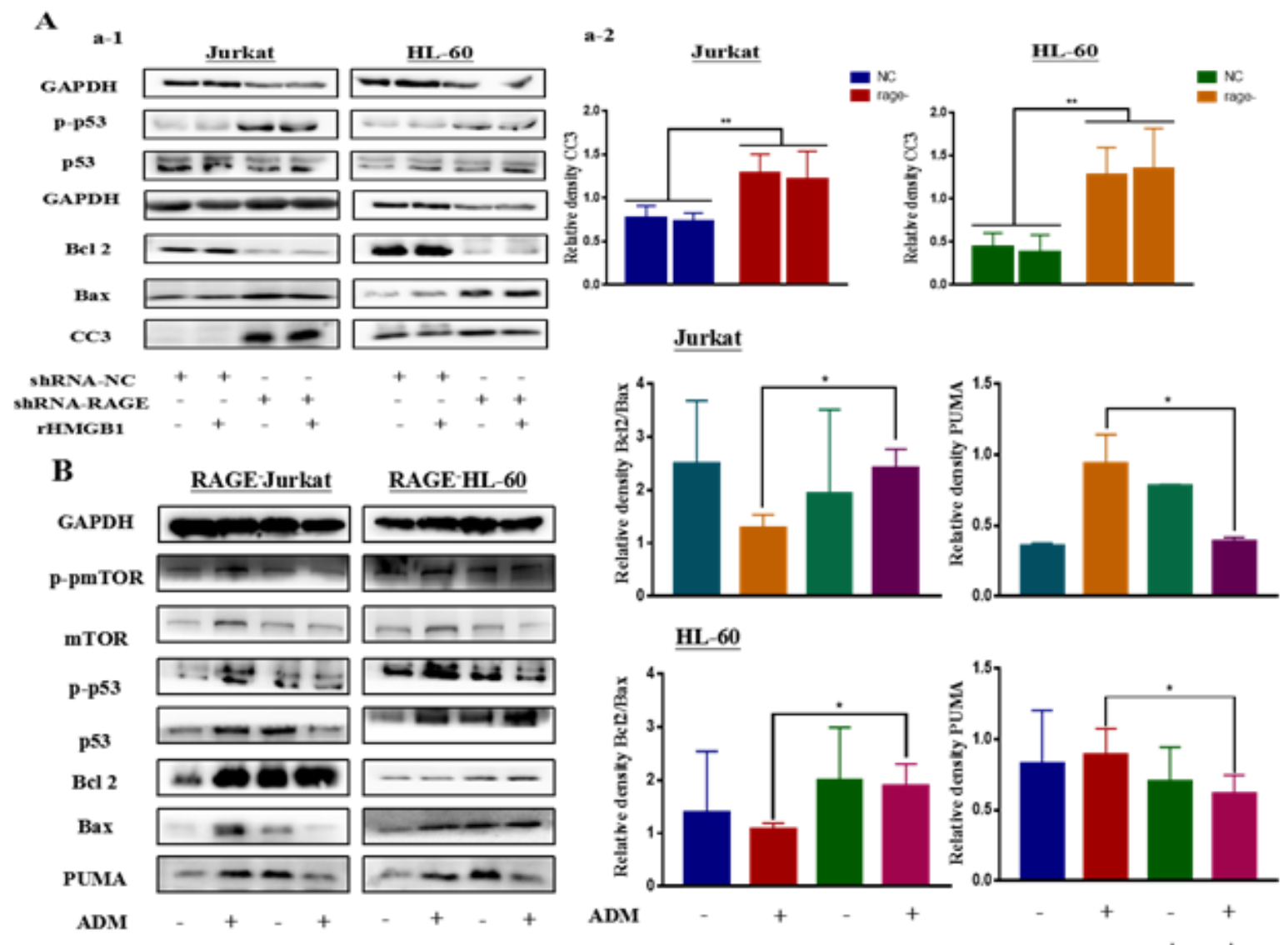

HL-60

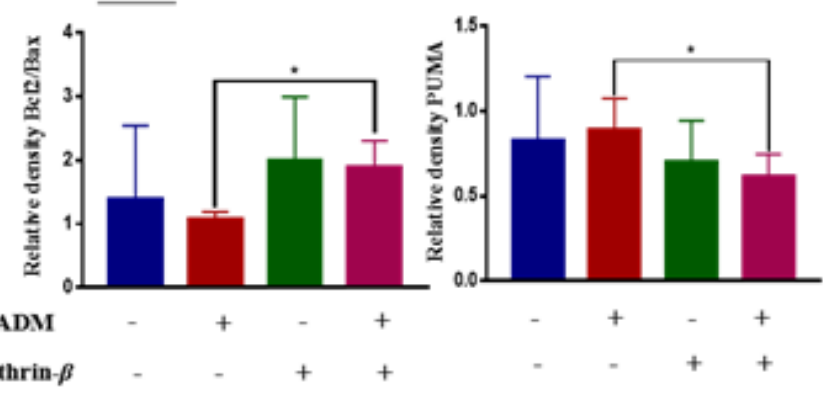

C
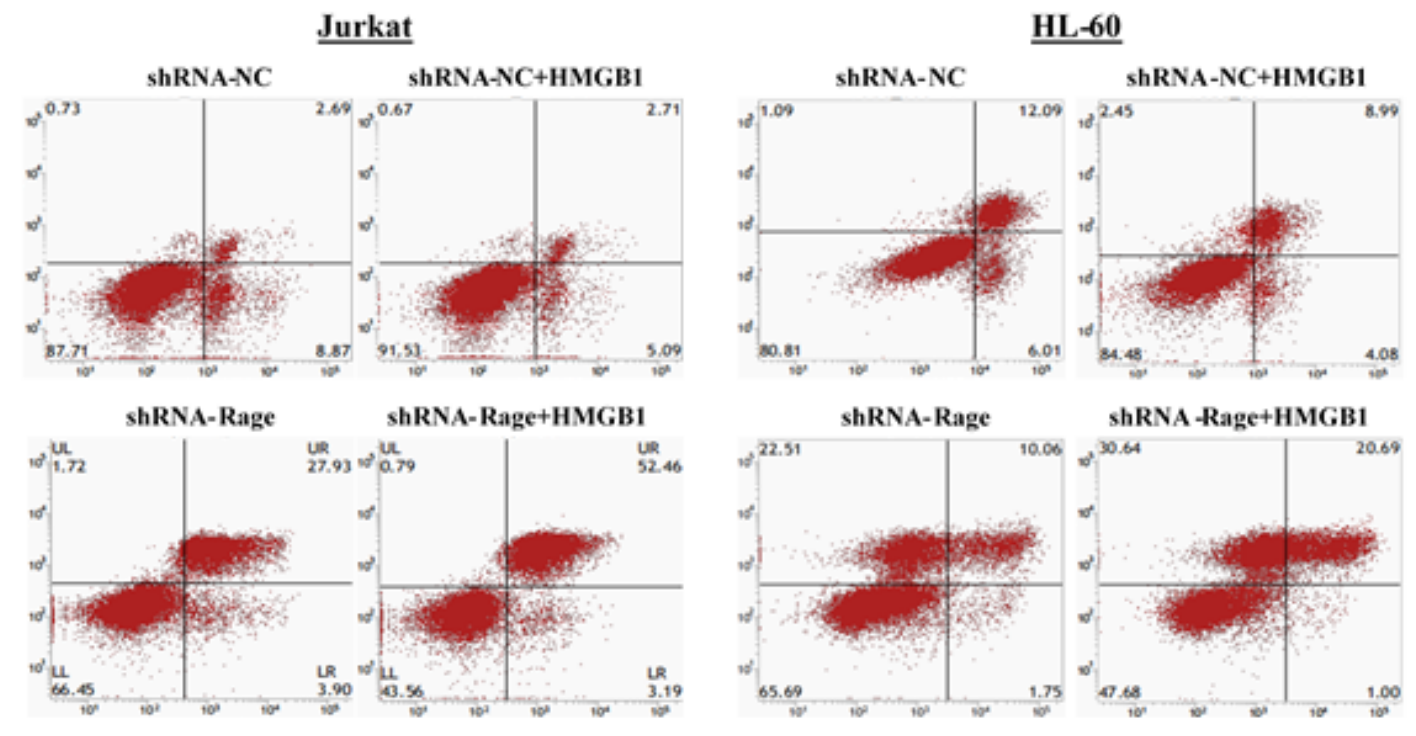

\section{Figure 4}

The ablation of RAGE enhanced apoptosis in leukemia cells and the apoptosis was related with the phosphorylated of P53. A (a-1) Cells that stably transfected with NC shRNA and RAGE shRNA were pretreated with $50 \mathrm{ng} / \mathrm{mL}$ rHMGB1 for $24 \mathrm{~h}$, then cells were co-cultured with $0.4 \mu \mathrm{M}$ ADM for another $24 \mathrm{~h}$. Western blotting was used to detect the alternation of apoptosis. (a-2) was the quantitative data of cleaved caspase 3/GAPDH. B (b-1) Cells that stably transfected with shRNA-RAGE were pretreated with 
the small inhibitor of $p 53$, Pifithrin $-\beta(1 \mu \mathrm{M})$ for $12 \mathrm{~h}$, following the treatment of $A D M(0.4 \mu \mathrm{M})$. Cells were subjected to western blotting to assess the level of apoptosis and the phosphorylation of P53. (b-2) were the quantitative data of Bcl 2/Bax and PUMA/GAPDH in both leukemia cells. (C) Cells in step A were collected and washed with PBS for 2 times. Annexin V APC-A and APC-Cy7-A were used to evaluate the level of apoptosis in different groups. Data are the mean \pm standard deviation of three independent experiments. ${ }^{*} \mathrm{P}<0.05,{ }^{*} \mathrm{P}<0.01$, compared with the ADM-treated NC group.
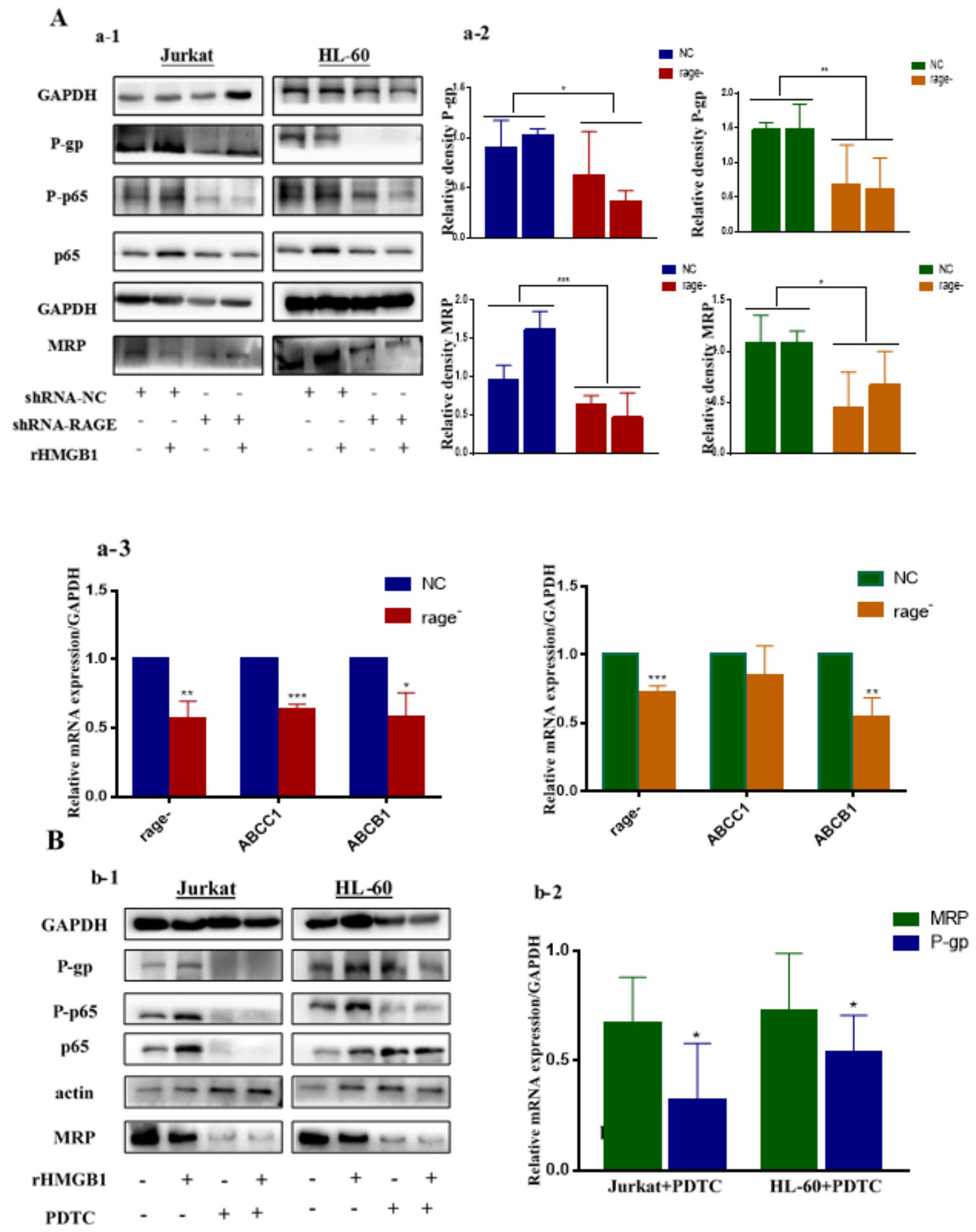

Figure 5 
HMGB1/RAGE induced the expression of drug resistance protein via NF-kB pathway. (A) (a-1) Leukemia cells that stably transfected with NC shRNA and RAGE shRNA were pretreated with $50 \mathrm{ng} / \mathrm{mL}$ rHMGB1 for $24 \mathrm{~h}$, then cells were co-cultured with $0.4 \mu \mathrm{M}$ ADM for another $24 \mathrm{~h}$. Western blotting was used to detect the expression of P-gp and MRP as well as the phosphorylation of P65.(a-2) was the quantitative data of Pgp and /GAPDH in both leukemia cell lines. (a-3)was the quantitative data of RAGE , ABCB1 and $A B C C 1 / G A P D H$ in mRNA expression. $B(b-1)$ Cells that stably transfected with shRNA-NC were pretreated with the small inhibitor of NF-KB, PDTC $(1 \mu \mathrm{M})$ for $12 \mathrm{~h}$, following the treatment of $A D M(0.4 \mu \mathrm{M})$. Cells were subjected to western blotting to assess the expression of P-gp and MRP and the phosphorylation of P65. (b-2) were the mRNA expression of P-gp and MRP of cells treated with PDTC compared to NC group.GAPDH was used as loading control. Data are the mean \pm standard deviation of three independent experiments. ${ }^{*} \mathrm{P}<0.05,{ }^{*} \mathrm{P}<0.01$ 
A
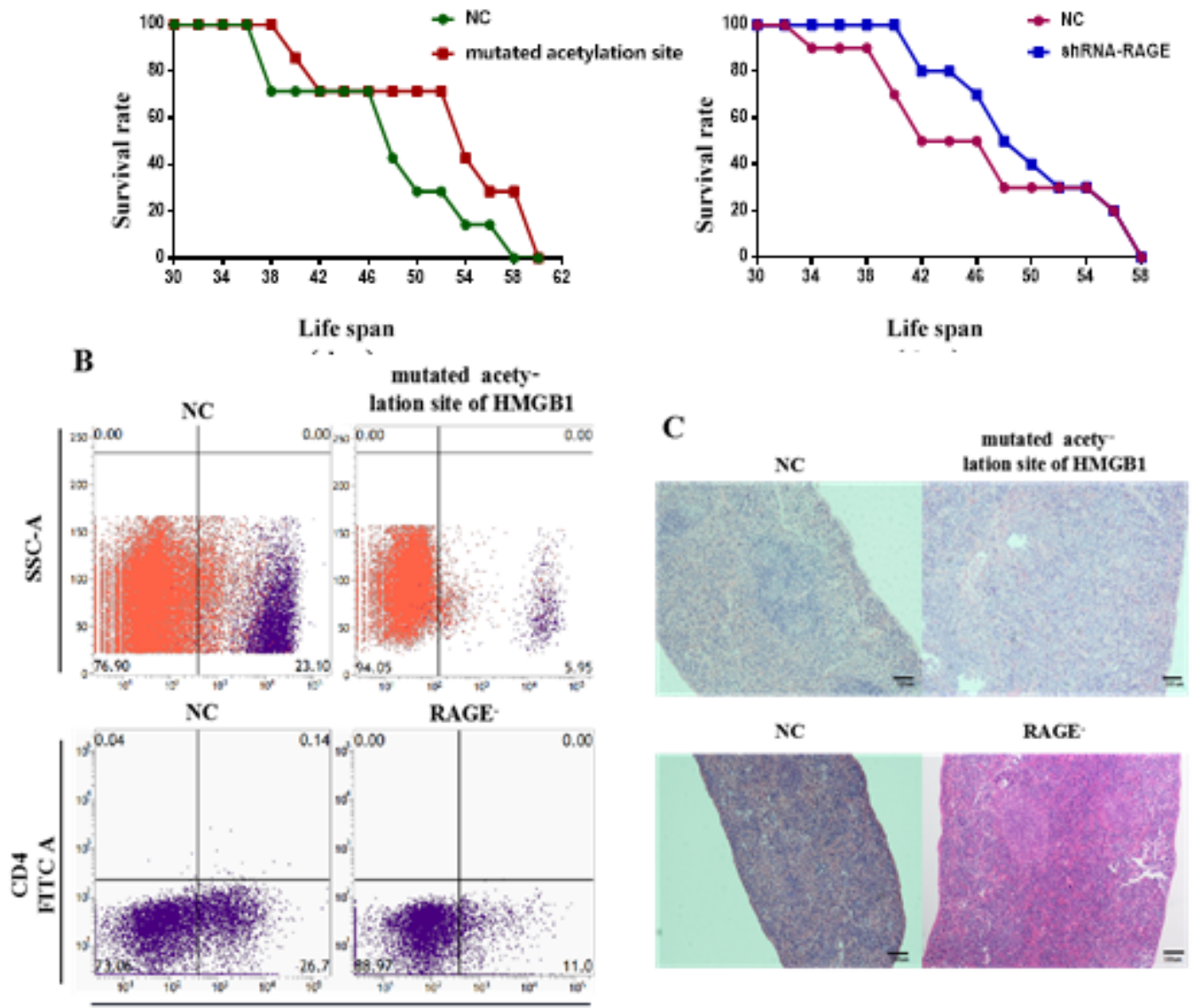

D

CD3 $\mathrm{V} 450 \mathrm{~A}$

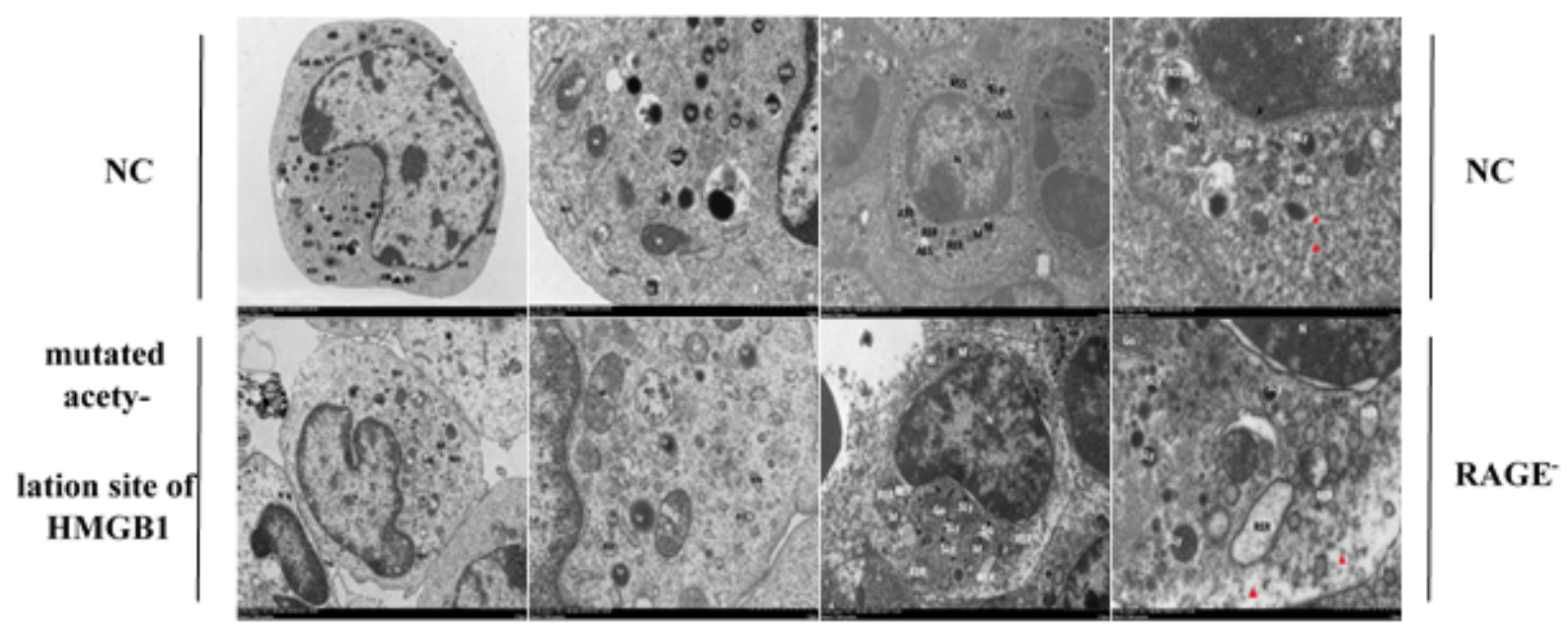

Figure 6

The obstruction of HMGB1/RAGE increased sensitivity to chemotherapy in vivo .(A) 5×106 Jurkat cells with stable transfection of control and Rage shRNA as well as mutated acetylation site of HMGB1 were injected into NOD/SCID mouse via intravenous injection to establish a model of leukemia mouse. Low dosage of $A D M(1 \mathrm{mg} /$ per mouse) was injected into mouse every other day. The diagram showed us the life span of mouse in different groups. B.Bone marrow sediment of mouse was removed and stained with 
BV421-CD3 before flow cytometry to detect the proportion of leukemia cells in bone marrow. C. When mouse come to the endpoint of death, spleen was separated and fixed with $4 \%$ paraformaldehyde. Pathological section was made by using hematoxylin-eosin staining. D. Bone marrow sediment of mouse was removed and subjected to transmission electron microscopy to observe autophagosomelike structures (indicated by red triangle).

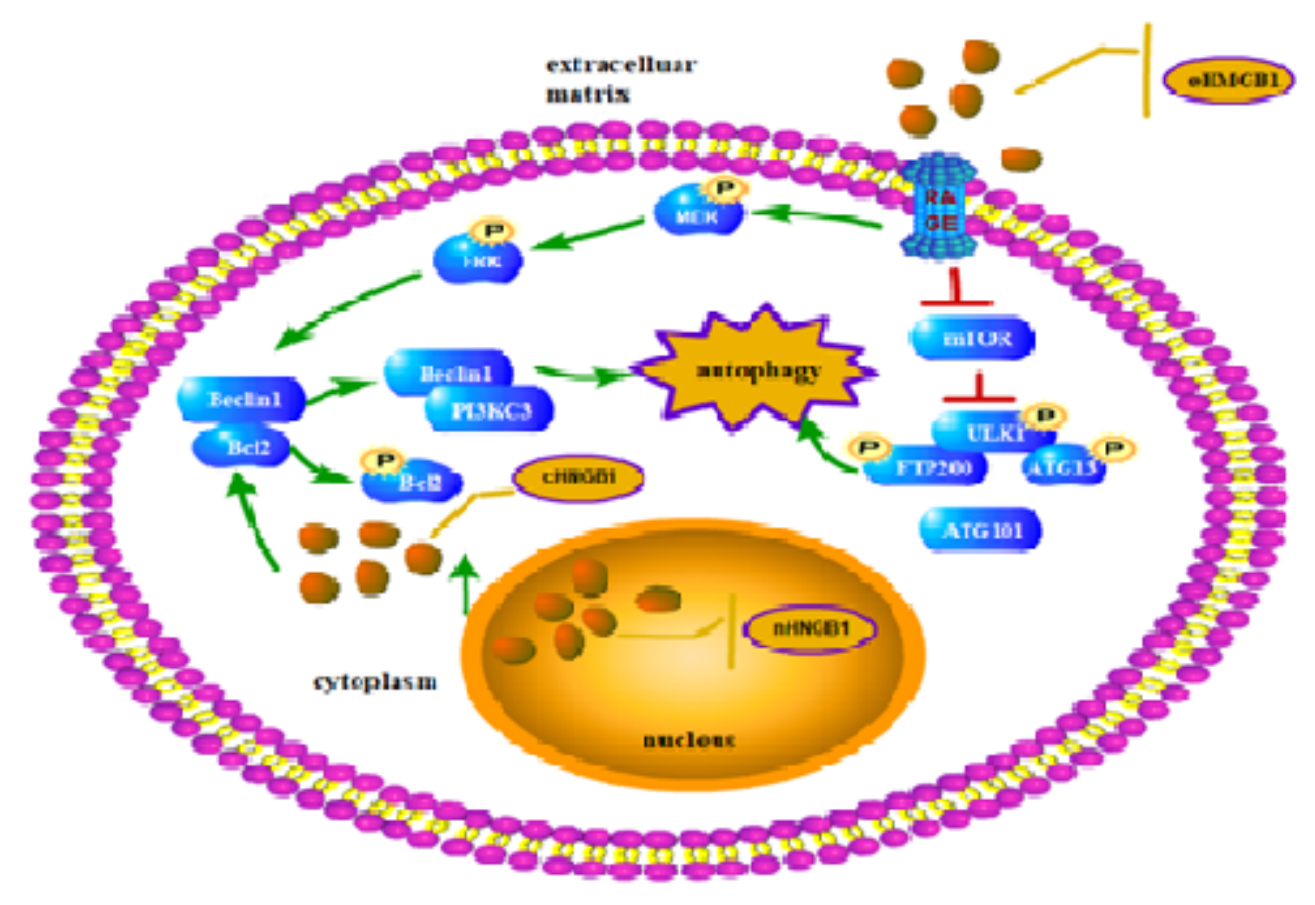

Figure 7

Schematic representation of the potential autophagy-related signaling pathway in leukaemia. Under stress condition, HMGB1 translocates to the cytoplasm and then release into the extracelluar matrix. Both cHMGB1 and eHMGB1 dissolocate the Beclin 1- Bcl 2 complex, facilitating the formation of PI3K3CBeclin 1, which leads to the initiation of autophagy. What's more, extracelluar HMGB1 can suppress the activation of mTOR and then phosphorylate the ULK1-ATG13-FIP200 complex and then promote autophagy. 